\title{
Service-Based Grid Resource Monitoring with Common Information Model
}

\author{
Hongyan Mao, Linpeng Huang, and Minglu Li \\ Department of Computer Science and Engineering, \\ Shanghai Jiaotong University,Shanghai 200030, China \\ \{mhy, lphuang, mlli\}@sjtu.edu.cn
}

\begin{abstract}
The monitoring of grid environments helps administrators and users keep track of the availability and loading of resources, and the management of resources is dependent on the monitoring of information data. There is not an efficient and consistent monitoring mechanism to the manipulation of devices, resources and services in Grid computing. We propose a novel monitoring framework used to gather and retrieve monitoring information of Grid environments. The monitoring system RMCS integrates and extends the existing monitoring system using service-oriented mechanism and the common information model CIM. The RMCS defines a hierarchical structure of monitoring resources, and customizes the monitoring parameters and the display way. The adoption of CIM-based monitoring service enables compatible with other grid services such as grid portal, transaction or resource management and charging. The investigation shows that this monitoring approach provides the scalable monitoring capabilities, enables to exchange information in an unrestricted and flexible way, and improves grid performance and utilization.
\end{abstract}

\section{Introduction}

Grid technology enables sharing and accessing distributed resources that how to manage the performance and status of these resources is a challenging research [1]. The management of resources is dependent on the monitoring of information data that displays every node status, historical cpu and memory latency and network loading. GMA specification defines the Grid monitoring architecture in terms of the producerconsumer model [2]. The Ganglia system gives the graphics mode for monitoring the $\mathrm{cpu}$, memory and loading performance of clusters and nodes [3]. M.A. Baker and G. Smith implement the monitoring prototype that provides the Grid sites map and the status of each site's MDS server [4]. The Vega project depicts the topology structure for the grid environments based on LDAP directory service [5]. There are limitations about the existed monitoring system and network. Administrators and users can only get and view the monitoring data in a fixed way, not customize the monitoring information according to domain-specific or user-specific favor. These approaches do not provide a general monitoring model that can be used in different scenarios and environments. We propose a novel monitoring strategy based on the common information model (CIM), which describes the overall management information of network and enterprise environment including devices, systems, applications. This monitoring 
system RMCS built on the services-oriented policy and CIM mechanism provides a standard and unified framework for monitoring logical and physical objects of grid environments. The RMCS organizes the monitoring object in a hierarchy structure that is scalable and extensible, building on which, we can set up the monitoring information according to diverse requirements.

Following the first section, section 2 briefly discusses the common information model. Section 3 elaborates the monitoring architecture, the representation of resources and the implementation mechanism. Finally, a short conclusion summarizes the work done and proposes the future research.

\section{Common Information Model}

The Common Information Model (CIM) is a model for describing information of network or enterprise environments [6]. An essential aspect of the CIM approach is the preservation and extension of traditional information resources. The CIM schema enables developers to describe monitoring data in a standard format so that it can be shared among a variety of applications. The CIM-XML encoding specification uses XML elements to represent CIM classes and instances. Aimed at particular grid environments, we design the schema of devices, system, network and applications by extending the core schema.

\section{The Service-Oriented Resource Monitoring with CIM}

\subsection{The Resource Monitoring Architecture}

In order to process raw information and efficiently analyze diverse network and applications, we propose a novel resource monitoring approach, which is based on CIM to describe and monitor information data. The architecture of the monitoring system RMCS is given in Fig.1.

There are many monitoring applications in grid and enterprise environments. The application generates requests to the CIM object management (CIMOM). The CIM$\mathrm{XML}$ is responsible for the request and response of monitoring messages between Client and CIMOM. The CIMOM parses and handles these requests using the schema repository, and deals with communications between applications and providers. In the schema, the information of monitoring environments is organized consisting of a set of classes, properties, methods, and associations. The schema is intended as a basis for platform and domain-specific extensions. The schema information is stored in the repository. When the request coming, CIMOM searches the corresponding schema in repository. The provider interacts with monitoring objects such as operating system, network and application, and retrieves the information data. The separation of the abstract service description from a corresponding service implementation enables providers to realize services according to the local environment without restricting or implying a particular implementation. 


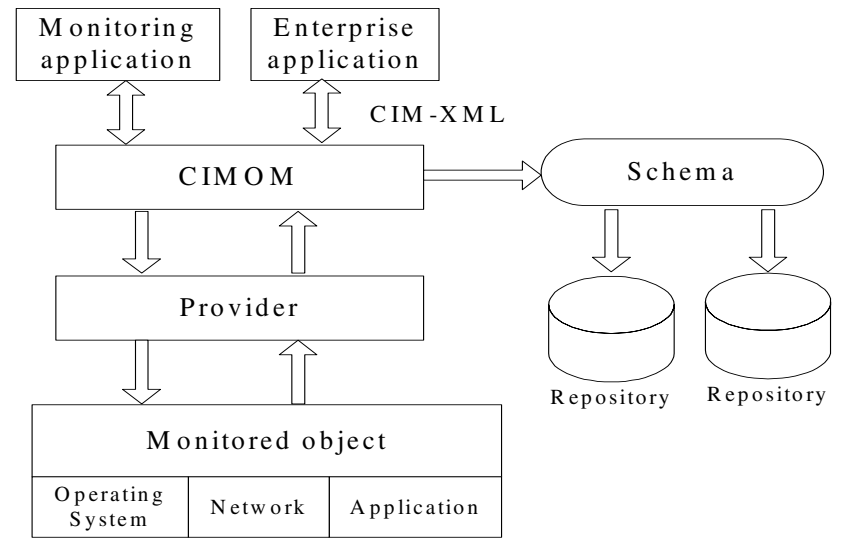

Fig. 1. The resource monitoring architecture

\subsection{The CIM Based Monitoring Resource Representation of Grid Environments}

In grid computing, we need to monitor and manipulate a large number of hardware and software components. Many resources possess the same properties and behaviors so that we adopt the hierarchical structure to depict grid elements. We use classes to describe resources, and associations to define the dependency and aggregation relationships between objects. Building on the common information model, the schema of every element is represented such as network card, disk, router and application. Every object is depicted using the Managed Object Format (MOF) description language [7, 8]. However CIM does not provide all the classes of various applications, so we have to extend CIM schema by adding new subclasses and associations with properties and methods to express the monitoring resources.

\subsection{The Mapping Between Monitoring Object and CIM Schema}

The operating system is investigated to show how to describe the monitoring object, and define the mapping from objects to CIM schema. The operating system is described a series of classes that Cpu, Memory and Process are subclass of Operating system. The Managed Object Format (MOF) files contain the definitions of class and instance, which are automatically imported into the CIMOM by management tool. The repository allows the extract from repository to MOF format containing usable monitoring data. CIMOM searches the registry information in the CIM schema repository.

\subsection{The Implementation of RMCS}

We have described the monitoring object and the schema, and then build the development in linux platform that uses Apache server as web server and Pegasus as CIM server. Pegasus is an open-source object manager for CIM objects. It includes the object manager, a set of defined interfaces, and SDKs for client, providers, and services extensions. The various monitoring services are automatically mapped to 
resource instrumentations. The provider returns the values of the monitoring component according to users' customized parameters and it contains a list of resources instances. The RMCS makes administrators and users define a wide variety of monitoring resources, specify the monitoring parameters and the display way.

\section{Conclusions}

We present a novel approach with CIM for the resource monitoring of grid environments. The resource monitoring system RMCS built on CIM and service is given that provides a general monitoring model for different scenarios and environments. It uses the hierarchical structure to define and represent the monitoring elements as classes and associations. The RMCS integrates the existing monitoring architecture, allows exchanging information in a flexible way. As well, the adoption of CIM-based monitoring service enables compatible with other grid services. In future, we will dedicate the perfection of the RMCS such as addressing the recovery of CIMOM failure, and apply to more grid and enterprise environments.

\section{References}

1. B. Bartosz, B. Marian Bubak, F. Włodzimierz et al. An infrastructure for Grid application monitoring. LNCS, Vol 24742002.

2. B.Tierney, R. Aydt, D. Gunter et al. A Grid Monitoring Service Architecture. Global Grid Forum White Paper, 2001.

3. M.L. Massie, B.N. Chun, D.E. Culler. The Ganglia Distributed Monitoring System: Design, Implementation, and Experience. Parallel Computing 30, pp 817-840, 2004.

4. M.A. Baker and G. Smith. A Prototype Grid-site Monitoring System. Version1. DSG Technical Report, January 2002.

5. http://www.vaga.com, VEGA GOS V1.1 manual.

6. Common Information Model (CIM) Specification. Distributed Management Task Force Version 2.2, 1999.

7. K. Alexander, K. Heather, S. Karl. Towards a CIM Schema for RunTime Application Management. 12th International Worshop on Distributed Systems: Operations and Management, France, 2001.

8. Specification for the Representation of CIM in XML Version 2.0. Distributed Management Task Force, July 1999. 